\title{
Exploiting the Cellular Infrastructure for Data Transmission in Smart Metering Systems
}

\author{
Athanasios Giannakopoulos, Panayotis G. Cottis \\ School of Electrical and Computer Engineering, National Technical University of Athens, Athens, Greece \\ Email: tgiannak91@gmail.com,pcottis@central.ntua.gr
}

Received 25 February 2016; accepted 22 May 2016; published 25 May 2016

Copyright (C) 2016 by authors and Scientific Research Publishing Inc.

This work is licensed under the Creative Commons Attribution International License (CC BY). http://creativecommons.org/licenses/by/4.0/

c) (i) Open Access

\begin{abstract}
To implement the access and backhaul networks for Smart Metering (SM) systems various technologies are combined with the existing communications infrastructure. This paper deals with data transmission in SM systems, focusing on how the existing cellular networks infrastructure is employed to implement SM access communication networks. The analysis aims at analyzing the role of the cellular communications infrastructure taking into account the spatial distribution and installation points of the smart meters, the urban and topological characteristics of the SM deployment areas and the common practice so far followed by the utilities. It is demonstrated that cellular communications, either exclusively or combined with power line communications, enable immediate and scalable deployment of SM access communication networks at low installation cost, thus constituting the basic option for the implementation of smart metering.
\end{abstract}

\section{Keywords}

Smart Metering (SM), SM Access Communication Networks, Cellular Communication Networks, Power Line Communications

\section{Introduction}

The Advanced Metering Infrastructure (AMI) enables the collection of billing data and critical measurements concerning the power grid based on automated two-way communication between Smart Meters (SMs) or Intelligent Electronic Devices (IEDs) and the utilities. Smart metering (SM) systems consist of three basic components: the SMs, the Meter Data Management System (MDMS) and the communications network connecting the SMs with the MDMS. The SMs are electronic end-devices that perform periodic electricity measurements (e.g. 
every 15, 30 or $60 \mathrm{~min}$ ) and record consumption of electric energy. At least once per day, the SMs transmit the collected data to the MDMS for power system monitoring and customer billing. The MDMS is responsible for storing and processing the SM data. If necessary, such as in emergency situations, the MDMS may send commands to specific SMs, e.g. for power supply activation/deactivation.

Several studies have been performed concerning the communications infrastructure and technologies related to SM data transmission. SM communication networks and their components, namely the SMs, the PLC concentrators, the base stations (BSs) of the cooperating cellular networks, hereafter referred to as serving BSs, and the MDMS are discussed in [1]. The communications technologies and standards related to smart metering are presented in [2], where communication technologies candidate for the SM access communications network, such as ZigBee, power line communications (PLC), cellular communications technologies, etc., are discussed and compared with regard to the services supported, the coverage and data rates offered, and the relevant performance limitations. The technical challenges that must be addressed by the SM communications infrastructure are examined in [3]. The commonly used PLC protocols are discussed and compared in [4]. [5] deals with the end-to-end communication between the SMs and the MDMS using either a combination of PLC and wireless/ cellular technologies or the latter option exclusively. The new services and functions offered by the Smart Grid (SG) for energy producers, distributors and consumers have led to the implementation of many pilot or large scale SM projects [6].

As analyzed in Section 2, the SM systems employ the cellular communications infrastructure for: 1) the collection of SM data, either directly from the SMs or indirectly from local PLC concentrators or 2) backhauling of SM data from the serving BSs to the MDMS. This work studies the suitability of cellular communications for the support of the SM access communications network, hereafter referred to as SM access network. The analysis takes into account the particular characteristics of the SM deployment areas and the operational features necessary for the implementation of the basic SM services. The density and installation points of the SMs in relation with the existing communications infrastructure are also taken into account.

To the best of the authors' knowledge, there has not been an analytical dimensioning of the SM access network using wireless technology. The relevant analysis, presented in this paper, shows that cellular networks, either exclusively or combined with PLC transmission via the LV grid, offer effective implementation of SM access networks as they provide immediate SM system deployment at low installation cost by exploiting the already existing infrastructure. Also, in addition to effectively supporting the communications of SM systems, the capacity offered by $3 \mathrm{G}$ or $4 \mathrm{G}$ cellular networks is found sufficient for the support of additional smart grid or smart home services. On the other hand, if the cellular implementation option of the SM access network is followed, the availability and capacity are subjected to unwanted variations induced due to the traffic variability of human-to-human $(\mathrm{H} 2 \mathrm{H})$ services which are primarily served by the cooperating cellular networks.

This paper is organized as follows. Section 2 provides an overview of SM systems and the communications technologies employed in the access and backhaul SM communication networks. Section 3 outlines the fundamental characteristics and specifications of SM access networks based on the best practices followed by the main European SM deployments. Section 4 presents an analytical dimensioning of SM access networks employing either direct SM data transmission from the SMs or from PLC concentrators. GPRS/3G-based SM access networks are analytically dimensioned in Section 5. Section 6 presents the key design Considerations for the SM access network. Finally, Section 7 concludes the paper.

\section{Overview of Smart Metering System}

SM systems are machine-to-machine (M2M) communications based systems that consist of the following components:

1) The SMs, which are end-devices responsible for performing electricity measurements and transferring it along with billing data first to local SM data concentrators and there from to the MDMS.

2) The local SM data concentrators, which collect the data acquired from the SMs and transmit it to the MDMS. The SMs around a local SM data concentrator form a neighborhood area network (NAN).

3) The MDMS, which is the information system of the utility. The MDMS is responsible for storing and processing the SM data. If required, the MDMS sends commands to specific SMs.

4) The SM communications network which consists of the access and the backhaul network. Depending on the overall SM system planning, the SM access network connects the SMs with the local SM data concentrators, 
usually the serving BSs, whereas the backhaul network connects the latter with the MDMS.

The two-way SM communications network must support reliable and secure data transfer between the SMs and the MDMS, characterized by low delay and adequate data rates. Depending on the population density and the urban development of the SM deployment area, the communications technology employed in the SM access network must provide: 1) ubiquitous coverage; 2 ) guaranteed availability; 3 ) scalable, stable and enduring (more than 20 years) operation; and 4) secure SM data transmission. A comprehensive discussion of the communications technologies destined for SM data transmission is provided in [7].

\subsection{The SM Access Communications Network}

The general structure of the SM communications network is depicted in Figure 1. The SM access network lies between the SMs and the serving BSs which usually act as local SM data concentrators. It is primarily destined for automated SM data collection and may employ either wireless or wired transmission. It can be implemented relying either on the existing or on a dedicated communications infrastructure, the former option being preferable in most cases. In fact, to implement SM data transmission in the access network of the majority of European SM deployments, PLC is combined with wireless/cellular technologies. Such a combination minimizes both the SM system implementation cost and roll out time as it employs the existing LV power grid and cellular communications infrastructure. Furthermore, such a combination offers almost 100\% coverage of the SM deployment area.

For the implementation of the SM access networks there are two alternatives. The first option is to employ the LV grid for PLC transmission of SM data (see App.1). The resulting SM access networks consist of two segments. The first PLC-based segment connects the SMs with local PLC concentrators. The second cellular-based segment connects the PLC concentrators with the serving BS. The other option is to transmit the SM data directly to the serving BSs, giving rise to one-segment SM access networks. That is, the serving BSs operate as local SM concentrators, either directly or indirectly as collectors of cumulative SM data from PLC concentrators.

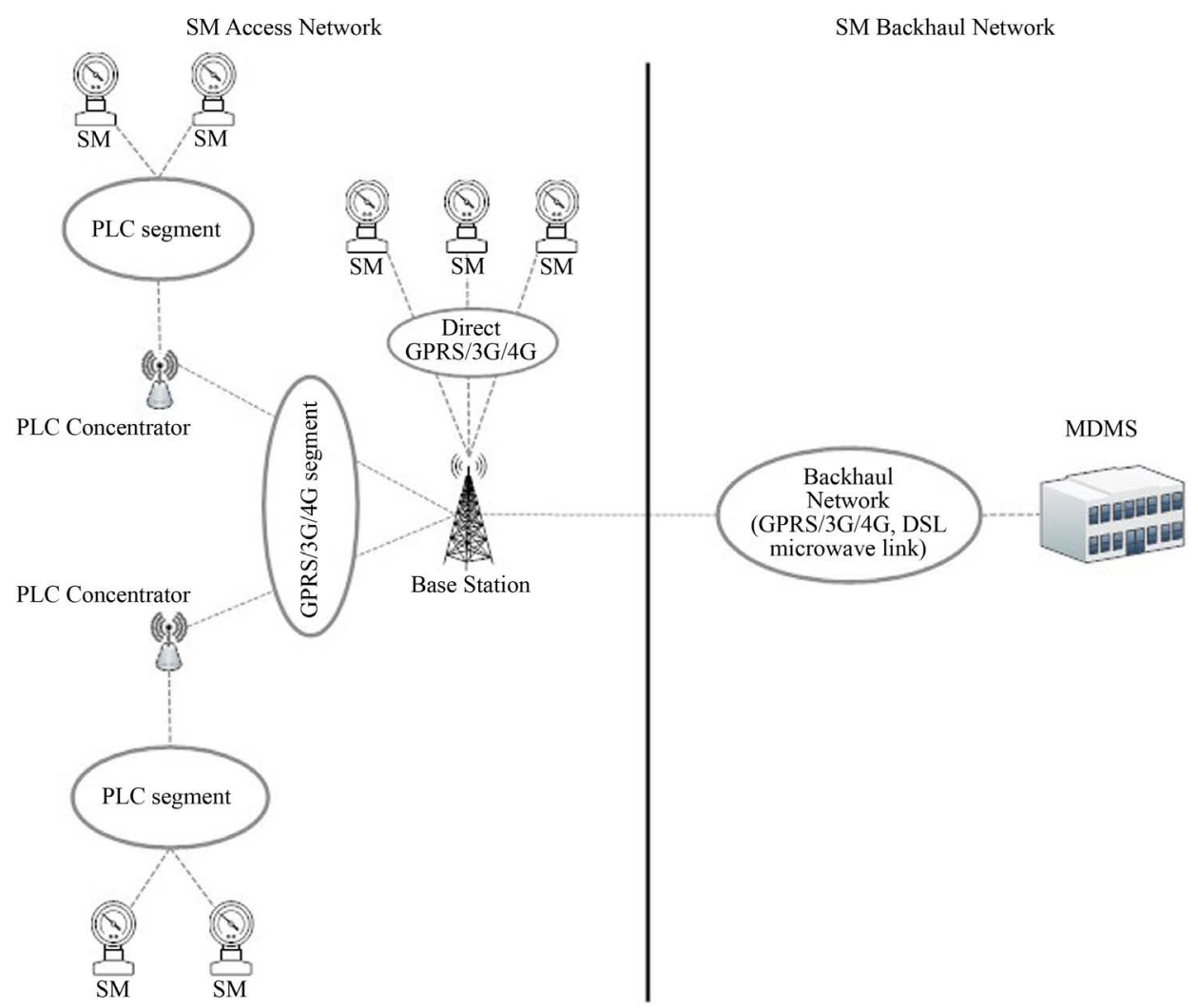

Figure 1. The SM access and backhaul networks. 


\subsection{The SM Backhaul Communications Network}

The SM backhaul communications network lies between the serving BSs and the MDMS. The backhaul network must provide: 1) significantly high data rates and ubiquitous coverage; 2) low end-to-end transmission delay and 3) reliable and secure data transmission.

In special cases, the SM data backhauling may be implemented employing DSL, point-to-point (P2P) microwave links or satellite communications. But, in most cases, the SM backhaul network is implemented employing the existing cellular communications infrastructure as it offers sufficient capacity, immediate deployment at low installation cost, and almost $100 \%$ coverage. On the other hand, apart from engaging mobile network operators as third parties, the availability and QoS of cellular based SM backhaul networks are affected by the variable traffic conditions related to the traditional $\mathrm{H} 2 \mathrm{H}$ services that are primarily served by the cellular networks.

As different communications technologies may be employed in the SM access and backhaul networks, interoperability is required. The interface between the access and the backhaul network must adapt the input data rate and packet structure to the output ones. Also, in the framework of supporting both real-time and loss-sensitive SM applications, the interface must be capable of assigning priorities to the SM data traffic.

\section{Basic Characteristics of SM Access Networks}

The successful implementation of SM access networks must meet two basic requirements. The first is related to the QoS levels specified for the SM services supported. The second is related to the necessity of matching the SM access network to the existing communications infrastructure. Hence, the SM topology is the basic factor determining the requisite SM characteristics. [7] and [8] deal with QoS assurance issues. [7] also studies the network architecture sad equate for specific SM deployment areas. The SMs may be either Full Function Devices (FFD) or Reduced Function Devices (RFD) [9]. A mapping of the available communications technologies to the various types of SM deployment areas, typically classified as sparsely populated, residential, and high-rising building areas, is attempted in [10] which takes into account specific evaluation criteria.

Many European countries have already implemented or are about to implement pilot or large scale SM projects [6]. In almost all European SM deployments, to transmit the SM data to the serving BSs a combination of PLC and wireless transmission is employed. The largest European SM project, deployed in Italy to serve approximately 30 million customers, employs PLC transmission to implement remote SM data reading and provide grid management services. The necessary PLC concentrators are installed at the local MV/LV substations and communicate with the MDMS via the cellular infrastructure. Sweden has implemented an SM project of smaller size that also employs PLC data transmission. Norway and the Netherlands plan SM projects following the Italian guidelines, considering also the deployment of gas and water smart metering systems. The gas and water SMs will communicate wirelessly with the electricity SMs, which will subsequently transmit the aggregate data to the nearest PLC concentrator. France has implemented an SM project for remote gas metering where the gas SMs communicate with the MDMS employing the serving BSs. SMs that are not covered by the local cellular networks engage repeaters. As the majority of pilot and large scale European electricity (and gas) SM projects use either exclusively wireless or two-segment PLC/wireless transmission, the advantages and disadvantages of each option are outlined in Table 1.

The design and implementation of SM access networks must primarily take into account 1) the spatial distribution and installation points of the SMs; 2) the existing cellular communications infrastructure; and 3) the technology appropriate for the specific SM access and backhaul network each time considered. Furthermore,

Table 1. Comparison of plc and wireless SM data transmission to the serving BS.

\begin{tabular}{|c|c|c|}
\hline & Advantages & Disadvantages \\
\hline$\underset{\mathcal{Q}}{u}$ & $\begin{array}{c}\text { The existing LV power grid is also used for SM data trans- } \\
\text { mission } \\
\text { Appropriate for densely populated urban areas }\end{array}$ & $\begin{array}{l}\text { Extra cost for PLC concentrators } \\
\text { Noisy transmission medium }\end{array}$ \\
\hline 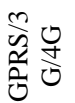 & $\begin{array}{l}\text { Immediate deployment } \\
\text { High data rates offered }\end{array}$ & $\begin{array}{c}\text { Extra cost for SM communications } \\
\text { Availability not always guaranteed } \\
\text { Dependence on third parties (the mobile network operators) }\end{array}$ \\
\hline
\end{tabular}


adequate QoS levels should be specified for: a) the availability of the SM access network; b) the minimum SM data rate; c) the acceptable end-to-end transmission delay; d) the response time for basic remote grid operations. Appropriate QoS levels should also be specified for the SM backhaul network.

Depending on the population density and assuming one SM per three inhabitants, three area types are typically considered with regard to the spatial SM distribution: 1) urban areas (SM density $>2000 \mathrm{SMs} / \mathrm{km}^{2}$ ), 2) suburban areas (SM density $\simeq 800 \mathrm{SMs} / \mathrm{km}^{2}$ ) and 3) rural $/$ sparsely populated areas (SM density $\simeq 10 \mathrm{SMs} / \mathrm{km}^{2}$ ). A proper combination of PLC and wireless technologies can effectively support SM transmission in almost any type of SM deployment area and offer high availability/reliability and sufficient communications capacity. Also, the SM roll-out time and installation cost are minimized since both options employ existing infrastructures for SM data transmission. Moreover, as argued in Section 5 and Appendix, in addition to efficiently serving the standard SM services, the capacity offered by GPRS, 3G or 4G (LTE) is sufficient for providing additional SG services.

A critical issue related to SM communications is related to the installation points of the SMs. It should be pointed out that the conventional electricity meters are usually installed at locations (usually basements) that may not be adequately covered by the local cellular networks. Hence, if the utilities choose to simply replace the conventional electricity meters with SMs avoiding to make any other changes, as it is common practice so far, two-segment SM access networks must be formed employing PLC concentrators.

In any case, the selection of the communications technology each time appropriate for the SM access network should be the result of thorough studies that take into account 1) the particular characteristics of the SM deployment area, such as the urban development, the distribution density and installation points of the SMs etc.; 2) the existing cellular infrastructure and 3) the particular operational features specified.

\section{Exclusively Wireless SM Access Networks}

The implementation of exclusively wireless SM access networks is differentiated based on whether the SM deployment area is adequately covered by a cellular network.

1) In the rather unusual case of areas not covered by some cellular network, a special purpose SM, having the role of master SM, should be capable of communicating with the MDMS. The rest of the SMs in the area, having the role of slave SMs, must communicate with the master SM. In such areas, NANs are formed by the slave SMs around their master. The latter is responsible for transmitting the cumulative SM data collected in its NAN to the serving BS or directly to the MDMS.

2) In the usual case where the SM deployment area is covered by a cellular network, the SMs communicate directly with the serving BS and there from with the MDMS. The SM data measured by the SMs every 15 min are locally stored and transmitted once per day to the serving BS and there from to the MDMS.

When wireless transmission from the SMs to the serving BS is employed, data routing can be either single-hop or multi-hop. If the serving BS-acting as a local SM concentrator-is located within the communication range of the SMs, SM data routing to the serving BS is single-hop. Otherwise, it is multi-hop, where the SMs covered by the serving BS relay the SM data originating from SMs that are not covered. Direct wireless SM data transmission to the serving BSs gives rise to cellular-based SM LANs around the serving BSs. As indicatively shown later in this section, typical cellular BSs can support any number of SMs installed within their coverage. Cellular based SM access networks are preferable in suburban and rural areas characterized by low shadowing levels and adequate cellular coverage. They might also be preferable in urban areas of low density population characterized by low-rising buildings.

In cellular based SM access networks, the SMs communicate with the serving BS once per day. In general, assuming a 4-byte representation per electricity measurement [13], the SM data packet containing the SM data volume created per SM per day plus the required overhead is given by Equation (1). Here, a sampling period of $15 \mathrm{~min}$ is assumed, i.e. $4 \times 24$ samples per day. The payload of the SM data packet is analyzed in Table 2 .

$$
P_{S M}=\text { Overhead }+4 \times 24 \times 40 \simeq 4 \mathrm{kB}
$$

Hence, the transmission delay is

$$
t_{d}=P_{S M} / R
$$

where $R$ is the uplink transmission rate of a wireless/cellular session and $P_{S M} \simeq 4 \mathrm{kB}$. The corresponding SM data volume assuming a 6-byte representation is $P_{S M} \simeq 6 \mathrm{kB}$. 
Table 2. SM data per electricity measurement.

\begin{tabular}{cc}
\hline Packet field & Number of bytes \\
\hline Incoming-outgoing active energy & $2 \times 4$ \\
Incoming-outgoing reactive energy & $2 \times 4$ \\
Voltage and current & $2 \times 4$ \\
Phase deviation & $3 \times 4$ \\
Energy flow direction & 4 \\
Cumulative SM data & 40 \\
\hline
\end{tabular}

As the total session duration ranges between $25 \mathrm{~s}$ and $50 \mathrm{~s}$ for GPRS and from $13 \mathrm{~s}$ to $25 \mathrm{~s}$ for 3G, the overall delay for the completion of a single (once per day) SM data transmission from an SM to the serving BS depends primarily on the session time of GPRS/3G/4G connections and not on the transmission delay induced by the actual SM data transmission which is comparably negligible. That is, the exact SM packet size is not decisive in determining the aggregate transmission delay as the latter is primarily determined by the total session time, which consists of the session installation/termination times plus the actual SM data transmission time, which is dramatically smaller. The relevant analysis concerning the once per day wireless transmission of cumulative SM data from PLC concentrators to the serving BS is similar. The only difference is that the corresponding transmission refers to data packets equal to

$$
P_{P L C}=\text { Overhead }+n \times 4 \times 24 \times 40 \simeq n \times 4 \mathrm{kB}
$$

where $n$ is the number of SMs served by the PLC concentrator. Though the PLC data packets transmitted to the serving BS from PLC concentrators are many times longer compared to the ones concerning individual transmissions from the SMs directly to the serving BS, the transmission delay induced by the actual SM data transmission is still negligible compared to the standard session time of GPRS/3G/4G connections.

\section{GPRS/3G Based SM Access Networks}

SM access networks relying solely on the local cellular infrastructure may employ GPRS/3G/4G technologies. Usually, the SMs transmit the SM data to the serving BS every day between 00:00 - 08:00 hours, when the traffic originating from $\mathrm{H} 2 \mathrm{H}$ services is quite low, leaving sufficient capacity available for SM data transmission. When the 3G or the 4G option is available, the WCDMA protocol is used for access to the common transmission medium, allowing the SMs in the area to simultaneously share the same radio channels without causing mutual interference. The number of SMs that can transmit simultaneously depends on the code length, the larger the number of SMs that transmit simultaneously, the smaller the transmission rate available per SM. 3G based SM access networks, even more 4G based ones, are characterized by significantly shorter session durations compared to GPRS based ones. Overall, if available, 3G/4G connections for SM access networks are preferable to GPRS connections because they can support simultaneous SM data transmissions of shorter duration from more SMs.

The indicative analysis that follows refers to 3G based SM access networks, pointing out that, if available, 4G based SM access networks offer significantly enhanced capacity and better operational features compared to 3G ones. Assuming that only one 3G channel is leased for SM purposes, the uplink rate offered by $3 \mathrm{G}$ systems is given by

$$
R_{U, 3 G}=960 \mathrm{kbps} /(n-1)
$$

Also, the number of users that may simultaneously use the same channel employing WCDMA is equal to

$$
N_{S, 3 G}=2^{n}, 2 \leq n \leq 8
$$

To provide an indicative dimensioning of 3G based SM access networks that allow for both Frequency Division Duplexing (FDD) and Time Division Duplexing (TDD), let us assume that 16SMs $(n=4)$ are simultaneously served, which results in an uplink transmission rate equal to $15 \times 16=240 \mathrm{kbps}$. On the other hand, the number of SMs that can be served during the SM data collection time is

$$
N_{3 G}=N_{S, 3 G} \times\left(T_{c} / T_{3 G}\right)
$$


where $T_{c}=8 \times 60 \times 60=28800 \mathrm{~s}(=8 \mathrm{~h})$ is the total duration of the SM data collection, $T_{3 G}$ denotes the mean duration of a $3 \mathrm{G}$ session and $N_{S, 3 G}$ is the number of SMs that can be simultaneously accommodated in a single 3G channel using WCDMA.As stated in Section 4, the SM data transmission depends on the session time of the GPRS/3G connections and not on the transmission delay induced by the actual SM data. Since $13 \mathrm{~s} \leq T_{3 G} \leq 25 \mathrm{~s}$ and $N_{S, 3 G}=16$, (6) yields

$$
18400 \leq N_{3 G} \leq 35400
$$

To determine which area types can be effectively served by 3G networks, the above result must be related to the respective SM densities. The indicative result of (7) shows that 3G cellular networks, even more 4G ones, can effectively support SM access networks in any area type.

As in many cases 3G/4G may not be available, SM access networks may have to rely on GPRS. A similar analysis shows that cellular networks offering only GPRS can also effectively support SM access networks in suburban and rural areas. However, due to the relevant long session times, GPRS might be problematic in supporting SM access networks in densely populated areas. Indicatively, the number of SMs that can be served by one GPRS channel is given by

$$
N_{G P R S}=N_{S, G P R S} \times\left(T_{c} / T_{G P R S}\right)
$$

where $T_{G P R S}$ is the duration of a GPRS session and $N_{S, G P R S}$ is the number of SMs that can be simultaneously accommodated in a single GPRS channel. For $N_{S, G P R S}=3,25 \mathrm{~s} \leq T_{G P R S} \leq 50 \mathrm{~s}$, (8) yields

$$
1700 \leq N_{\text {GPRS }} \leq 3400
$$

The above result shows that in densely populated urban areas, leasing more channels might be necessary for the implementation of exclusively wireless SM access networks, consequently increasing the cost for cellular services.

\section{Key Design Considerations}

From the preceding analysis, the following key design considerations for SM access networks come up.

\subsection{Which Technologies Are Suitable for Data Transmission in SM Access Networks?}

If wireless connection with the SMs is hindered or when preferred by the utilities for practical reasons, the communication of the SMs with the serving BS is two-hop. The first hop connects the SM to the relevant local PLC concentrators employing the LV grid whereas the second hop connects the PLC concentrators with the serving BSs. If wireless SM connectivity is feasible, once per day direct wireless SM data transmission to the serving BS is preferable. 3G cellular networks, even more 4G (LTE) ones, can effectively support SM access communications in any area type. One $3 \mathrm{G}$ or $4 \mathrm{G}$ channel is more than sufficient for SM purposes. In densely populated areas where only GPRS connectivity is available, two GPRS channels might be necessary for SM purposes.

\subsection{Should There Be a Dedicated Communications Infrastructure for the SM Access Network?}

A dedicated infrastructure is not necessary since the wireless/cellular infrastructure either by itself or combined with PLC can provide full coverage and sufficient communications capacity for the implementation of SM access networks. Employing the existing infrastructure offers both minimum deployment time and implementation cost.

\subsection{Which Factors Determine the Communications Technology Each Time Preferable?}

The communications technology each time preferable for the SM access network depends on: 1) the population density, the urban development and the terrain profile of the SM deployment area; 2) the capacity available for SM purposes by the local cellular infrastructure; and 3) the QoS specified for the various SM services. Regarding the transmission of SM data from the SMs to the serving BSs, the exclusively wireless option is preferable in 
areas where either the LV power lines are long or wireless transmission is not severely impeded by multipath or shadowing. On the other hand, the PLC option is preferable in areas inadequately covered by the local cellular infrastructure or in areas where the LV grid consists of power lines of small length (see Appendix). Another reason of the utilities preference for the PLC option is that it requires minimum changes to the existing electrical infrastructure in order to replace the conventional electricity meters with SMs.

\subsection{What Is the Tradeoff between the One-Segment Cellular Based and the Two-Segment PLC Based Implementation of the SM Access Network?}

Whenever necessary or preferred by the utilities, the partially wired (PLC based) implementation leads to SM access networks comprising PLC segments structured around MV/LV substations. These PLC segments are deployed at a rather small installation cost for purchasing, installing and configuring the necessary PLC concentrators. As these PLC segments are owned by the utilities, the dependence of the latter on local cellular operators is reduced. Whenever feasible, the exclusively wireless (GPRS/3G/4G cellular based) implementation of SM access networks offers immediate installation at almost no cost. But, apart from rendering the SM access network fully dependent on third parties, this option leads to increased cost for communications services.

\subsection{Can the SM Access Network Support Additional Services?}

Both implementation options of SM access networks, especially the exclusively wireless one, are capable of providing sufficient capacity to support additional smart grid services and/or smart home applications. The idea of adding real time services should also be examined and could be the subject of future research work in the field of smart grid.

\section{Conclusion}

Cellular networks, either standalone or combined with PLC transmission via the LV grid, offer effective implementation of SM access communication networks, as they provide immediate SM system roll-out at low installation cost. In addition to effectively supporting the communications of SM systems, the capacity offered by 3G or $4 \mathrm{G}$ cellular networks is also sufficient for the support of additional smart grid or smart home services. The availability and capacity offered for SM purposes are subjected to variations induced due to the traffic variability of the $\mathrm{H} 2 \mathrm{H}$ services which are primarily served by the cooperating cellular networks.

\section{References}

[1] Narsingh Sahu, V.D. (2012) Intelligent Machine to Machine Communication in Home Area Network for Smart Grid. IEEE, ICCCNT, Coimbatore, 26-28 July 2012, 1-6.

[2] Güngör, V.C., Sahin, D., Kocak, T., Ergüt, S., Buccella, C., Cecati, C. and Hancke, G.P. (2011) Smart Grid Technologies: Communication Technologies and Standards. IEEE Transactions on Industrial Informatics, 7, 529-539.

[3] Yan, Y., Qian, Y., Sharif, H. and Tipper, D. (2013) A Survey on Smart Grid Communication Infrastructures: Motivations, Requirements and Challenges. IEEE Communications Surveys \& Tutorials, 15, 5-20.

[4] Hoch, M. (2011) Comparison of PLC G3 and PRIME. IEEE International Symposium on Power Line Communications and Its Applications, Udine, 3-6 April 2011, 165-169.

[5] Enexis (2013) G3-PLC at Enexis: Description of G3-PLC Technology and Pilot Results at Enexis. Enexis.

[6] Cervigni, G. and Larouche, P. (2014) Regulating Smart Metering in Europe: Technological, Economic and Legal Challenges. CERRE.

[7] Gungor, V.C. and Lambert, F.C. (2006) A Survey on Communication Networks for Electric System Automation. Computer Networks, 50, 877-897. http://www.sciencedirect.com/science/article/pii/S1389128606000193

[8] Kuzlu, M., Pipattanasomporn, M. and Rahman, S. (2014) Communication Network Requirements for Major Smart Grid Applications in HAN, NAN and WAN. Computer Networks, 67, 74-88. http://www.sciencedirect.com/science/article/pii/S1389128614001431

[9] Farahani, S. (2011) ZigBee Wireless Networks and Transceivers. Newnes.

[10] T. E. P. Company (2012) Basic Specifications for Smart Meter Communications.

[11] Fernandes, A.D.L. and Dave, P. (2011) Power Line Communication in Energy Markets. 
http://www.cypress.com/file/109236/download

[12] 3G Cellular Technology for Smart Grid Communications. Qualcomm, 2011.

[13] E.C. Limited (2010) High-Level Smart Meter Data Traffic Analysis. 


\section{Appendix: PLC Transmission to the Serving BS}

To minimize the changes in the existing electrical infrastructure required to replace the conventional electricity meters with SMs and, at the same time, exploit the existing LV grid for communication purposes, the utilities seem, so far, to prefer employing PLC technology for SM data collection in the SM access network. Such a preference is more pronounced in urban areas where the LV power lines have small length, thus inducing small attenuation and negligible distortion. The PLC option is also adopted in SM deployment areas where wireless SM connectivity is hindered [12]. Specifications of narrowband PLC standards are tabulated in [11] [Table 3]. The data rates offered by the established PLC protocols are tabulated in [11] [Table 1].

PLC based SM access networks consist of two segments. The first PLC based segment lies between the SMs and PLC concentrators installed at local MV/LV substations where the SM data is stored until its wireless transmission to the serving BS on a daily basis. The second cellular based segment lies between the PLC concentrators and the serving BSs.

Along every LV power line stemming from an MV/LV substation, a bus topology is formed for the communication of the SMs with the serving PLC concentrator. Overall in a PLC segment, a star topology is formed by the LV power lines (typically 5 to 10) stemming from every MV/LV substation. The installation points of PLC concentrators must guarantee backhauling of the SM data to the MDMS, usually via the existing cellular infrastructure.

The parameters characterizing PLC based SM access networks in urban areas are tabulated in Table 3. Based on Table 3, it is found that the time required in a typical urban SM NAN for a transmission of the SM data collected from $n_{i}$ SMs fed by LV transmission line $i$ stemming from the MV/LV substation is equal to $t_{i}=900 \times\left(n_{i} / N_{t}\right)$. Though TDMA and CSMA/CD are both capable of supporting data routing in the PLC segment, the CSMA/CD option is preferable since it allows the incorporation of new SMs without requiring timeslot rescheduling.

In case simultaneous transmissions by two or more SMs are initiated along the same LV power line, a collision is detected by the CSMA/CD protocol and the SM data transmissions involved are postponed until the transmission medium is found vacant. Though, in general, the adoption of CSMA/CD reduces complexity at the expense of increased aggregate transmission delay, in PLC based SM access networks such a delay is negligible.

On the other hand, in order to implement collision detection, the CSMA/CD protocol imposes a minimum packet size

$$
P_{\min , i}=2 \times L_{i} / c_{\text {wire }} \times R_{S M}
$$

where $c_{\text {wire }}\left(\simeq 2 \times 10^{8} \mathrm{~m} / \mathrm{s}\right)$ is the speed of light in LV power lines. Since $500 \mathrm{~m} \leq L_{i} \leq L_{\max }=1000 \mathrm{~m}$, $\max _{i}\left\{P_{\min , i}\right\}=2 \times 10^{3} /\left(2 \times 10^{8}\right) \times 4.8 \times 10^{3} \simeq 0.0481$.

Hence, the CSMA/CD performance in PLC-based SM access networks is not constrained by a minimum packet size. On the other hand, the maximum extra delay induced when CSMA/CD is employed, is

$$
D_{e x}=e \times 2 \times L_{\text {max }} / c_{\text {wire }} \simeq 10^{-5} \mathrm{~s},
$$

which is negligible.

Table 3. Parameters for urban PLC-based SM access networks.

\begin{tabular}{ccc}
\hline Notation & Parameter description & Typical Values \\
\hline$N_{t}$ & Total number of SMs in a NAN & $\leq 350$ \\
$T_{P L C}$ & SM data collection period & $15 \mathrm{~min}(900 \mathrm{~s})$ \\
$k$ & Mean number of LV power lines per urban MV/LV substation & $\bar{k}=7$ \\
$n_{i}$ & Mean number of SMs per LV power line & $\bar{n}=50$ \\
$R_{S M}$ & SM data transmission rate & $4.8 \mathrm{kbps}$ \\
$P_{P L C}$ & Packet size & $192 \mathrm{~B}$ \\
$L_{i}$ & LV power line length & $500 \mathrm{~m} \leq L_{i} \leq 1000 \mathrm{~m}$ \\
\hline
\end{tabular}


Though the aggregate end-to-end delay is longer due to packet processing and signaling at the SMs and the PLC concentrators, these additional delays are practically negligible too. Hence, when employed in PLC based SM access networks, CSMA/CD offers reduced complexity and enhanced scalability at practically no extra delay.

Concluding, as urban LV power lines are short, SM data transmission in the PLC segment of urban SM access networks employing CSMA/CD is always feasible and induces negligible transmission delay. Moreover, since SM data acquisition from all the SMs in a PLC segment by the corresponding PLC concentrator is completed in drastically less than 15 min (900 s), additional real time smart grid services may be offered. 